\title{
Alexandre M.J.J. Bonvin \\ Localisation and dynamics of sodium counterions around DNA in solution from molecular dynamics simulation
}

Received: 12 August 1999 / Revised version: 11 November 1999 / Accepted: 7 December 1999

\begin{abstract}
The localisation and dynamics of sodium counterions around the DNA duplex d(AGCGTACTAGTACGCT $)_{2}$ corresponding to the trp operator fragment used in the crystal structure of the half site complex has been studied by a $1.4 \mathrm{~ns}$ molecular dynamics simulation in explicit solvent. A continuous and well-defined counterion density is shown to be present around the minor groove, while density patches are found in the major groove in regions where DNA bending is observed. A residence time analysis reveals the dynamic nature of these distributions. The resulting picture agrees with previous theoretical and experimental studies of A-tract DNA sequences, and is consistent with the polyelectrolyte condensation model.
\end{abstract}

Key words DNA - Molecular dynamics simulation . Sodium counterions $\cdot$ Localisation · Dynamics

\section{Introduction}

The traditional picture of DNA solvation in which the first few layers of solvation are exclusively occupied by water molecules is currently being replaced by a more complex view in which both water molecules and counterions partially occupy the first solvation shells. Counterions binding in the DNA grooves have been recently put in evidence both by computational (Young et al. 1997; Lyubartsev and Laaksonen 1998) and experimental techniques (Shui et al. 1998; Hud et al. 1999). These studies have concentrated on A-tract DNA

A.M.J.J. Bonvin

Bijvoet Center for Biomolecular Research,

NMR Spectroscopy, Utrecht University,

Padualaan 8, $3584 \mathrm{CH}$ Utrecht, The Netherlands

e-mail: abonvin@nmr.chem.uu.nl

Supplementary material to this paper (Fig. 1 in colour) can be obtained electronically using the Springer Link server located at http://link.springer.de/link/service/journals/00249/index.htm sequences because of their bending properties and a model for DNA bending based on an electrostatic collapse around bound counterions has been proposed (Rouzina and Bloomfield 1998; Shui et al. 1998; Hud et al. 1999). Here, the localisation and dynamics of sodium counterions around the DNA duplex d(AGCGTACTAGTACGCT) 2 corresponding to the trp operator fragment used in the crystal structure of the half site complex (Lawson and Carey 1993) [PDB (Bernstein et al. 1987) entry 1TRR] has been studied from a $1.4 \mathrm{~ns}$ molecular dynamics (MD) simulation in explicit solvent. The counterion density around the DNA is shown to be localised around the minor and major groove: a continuous and well-defined density is present around the minor groove while density patches are found in the major groove in regions where DNA bending is observed. The counterions keep, however, a dynamic character, as revealed from a residence time analysis. The presented results are consistent with the electrostatic collapse DNA bending model.

\section{Computational methods}

A MD trajectory previously generated for studying the dynamic of hydration of this DNA sequence (Bonvin et al. 1998) was used in this work. The MD simulation was run for $1.4 \mathrm{~ns}$ at constant temperature $(277 \mathrm{~K})$ and pressure (1 atm) using the GROMOS96 MD program and force field (van Gunsteren et al. 1996; Scott et al. 1999) starting from a B-form DNA (Arnott et al. 1976). The DNA duplex was solvated in a rectangular box of single point charge (SPC) water (Berendsen et al. 1981) with a minimum solute-wall distance of $1.4 \mathrm{~nm}$. Thirty sodium ions were added to neutralise the system, and 15 additional sodium chloride ion pairs were introduced to bring the system to an approximate $0.2 \mathrm{M}$ salt concentration. The ions were added one by one in an iterative manner by calculating the electrostatic potential of each water molecule and replacing the water molecule with 
Table 1 Interaction parameters $C_{12}(i, j)$ and $C_{6}(i, j)$ for sodium and chloride ions for the van der Waals interaction $C_{12}(i, j) / r_{i j}{ }^{12}-C_{6}(i, j) /$ $r_{i j}{ }^{6}$ between atoms $i$ and $j$ of the same type. The parameters for different types of atoms follow from $C_{12}(i, j)=\left[C_{12}(i, i) C_{12}(j, j)\right]^{1 / 2}$ and $C_{6}(i, j)=\left[C_{6}(i, i) C_{6}(j, j)\right]^{1 / 2}$

\begin{tabular}{lll}
\hline Atom $i$ & $\begin{array}{l}C_{6}(i, i) \\
\left(\mathrm{kJ} \mathrm{mol}^{-1} \mathrm{~nm}^{6}\right)\end{array}$ & $\begin{array}{l}C_{12}(i, i) \\
\left(\mathrm{kJ} \mathrm{mol} \mathrm{nm}^{12}\right)\end{array}$ \\
\hline $\mathrm{Na}$ & $7.2059 \times 10^{-5}$ & $2.1014 \times 10^{-8}$ \\
$\mathrm{Cl}$ & $1.3804 \times 10^{-2}$ & $1.0691 \times 10^{-4}$ \\
\hline
\end{tabular}

the most negative or positive potential by a $\mathrm{Na}^{+}$or $\mathrm{Cl}^{-}$ ion, respectively. The sodium and chloride ion nonbonded parameters used in this work are listed in Table 1 and correspond to the standard GROMOS96 force field parameters (Scott et al. 1999). The final system comprised 25522 atoms, including 718 DNA atoms, 60 ions and 8248 water molecules. The time step used in the leapfrog integration scheme was 0.002 ps. Covalent bond lengths were constrained with the procedure SHAKE (Ryckaert et al. 1977) with a relative geometric tolerance of 0.0001 . The temperature and pressure of the system were maintained by weak coupling $\left(\tau_{\mathrm{T}}=0.1 \mathrm{ps}\right.$; $\left.\tau_{\mathrm{P}}=0.5 \mathrm{ps}\right)$ to external temperature and pressure baths (Berendsen et al. 1984). The non-bonded interaction pair list was updated every five steps with a cutoff of $0.8 \mathrm{~nm}$. Non-bonded interactions were calculated with group cutoffs using the twin-range method (van Gunsteren and Berendsen 1990) with cutoffs of 0.8 and $1.4 \mathrm{~nm}$, respectively, and a reaction field correction to the atomic forces (Tironi et al. 1995). The last nanosecond of the trajectory (0.4-1.4 ns) was used for analysis. For additional details of the simulation see Bonvin et al. (1998).

\section{Results and discussion}

The counterions localisation around the DNA was studied by calculating an average sodium probability map in the same manner as was done for the hydration study of this DNA sequence (Bonvin et al. 1998). No chloride probability map was calculated because of the lack of ions in proximity to the DNA. The sodium probability map (analogous to an electron density map, DeLano and Brünger 1994) was calculated with a modified version of X-PLOR (Brünger 1993) from 1000 snapshots taken at $1 \mathrm{ps}$ interval from the last $1 \mathrm{~ns}$ of the MD trajectory (0.4-1.4 ns). This was done by superimposing the non-hydrogen atoms of the central six DNA base pairs onto the average structure and defining a three-dimensional unit cell enclosing these structures with a grid spacing of $0.03 \mathrm{~nm}$ and a cushion of $1.0 \mathrm{~nm}$. The probability density was calculated using standard crystallographic techniques from the sodium ions positions within the defined unit cell (for details, see Bonvin et al. 1998). The standard atomic scattering factors for oxygen were used in the calculations to allow a direct comparison with the water probability map calculated in a)
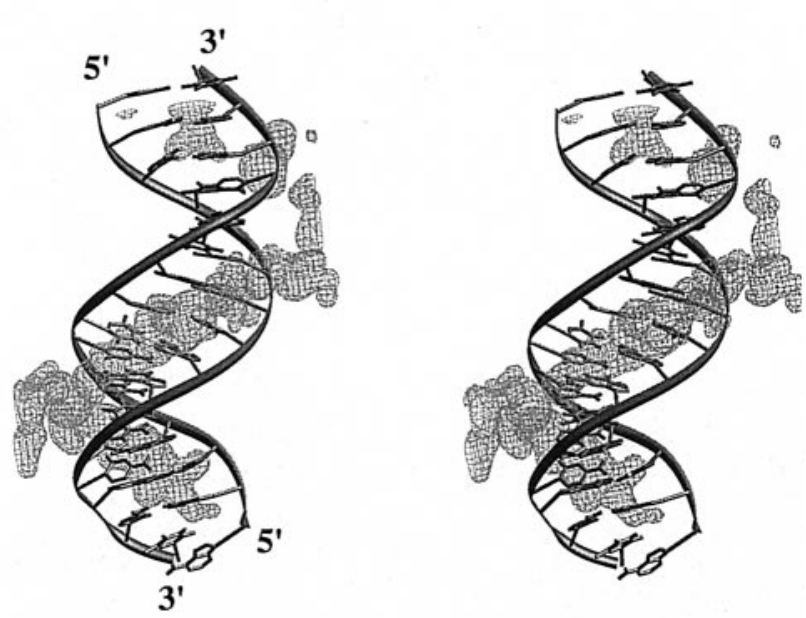

b)
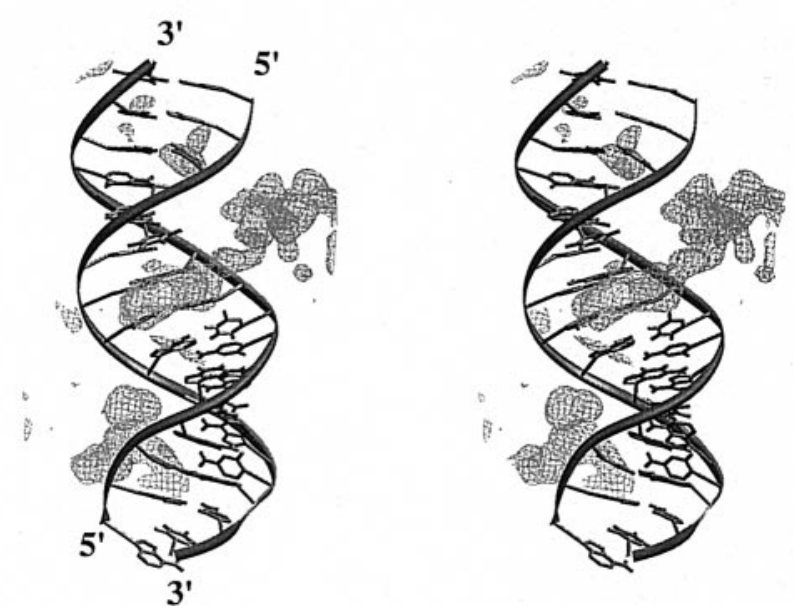

Fig. 1 Stereoviews of the sodium probability map around the average DNA structure: a minor groove, $\mathbf{b}$ major groove. The probability map was calculated from 1000 snapshots taken at 1 ps intervals from the 0.4-1.4 ns MD trajectory (see text) and is plotted at 2.5 standard deviations above the mean. The figure was generated with SETOR (Evans 1993)

the previous hydration study (Bonvin et al. 1998). The resulting probability maps, plotted at 2.5 standard deviations above the mean, are shown in Fig. 1 separately around the major and minor grooves. Continuous probability density is found in the minor groove, running approximately in between opposite phosphate groups. This is in agreement with the sodium ion distributions calculated by Lyubartsev and Laaksonen (1998). Discontinuous density is however found in the major groove where the density "patches" are approximately centred around DNA bending regions corresponding to pyridimine-purine steps. A probability map calculated from the last $0.5 \mathrm{~ns}$ of the trajectory shows similar features (see Supplementary material, Fig. S1), indicating that the calculated sodium ion density is reasonably well converged. The local bending is illustrated by the plot of the average roll parameters describing the angle between successive base-pair planes as a function of the DNA sequence in Fig. 2. The localisation around bending regions is consistent with the 


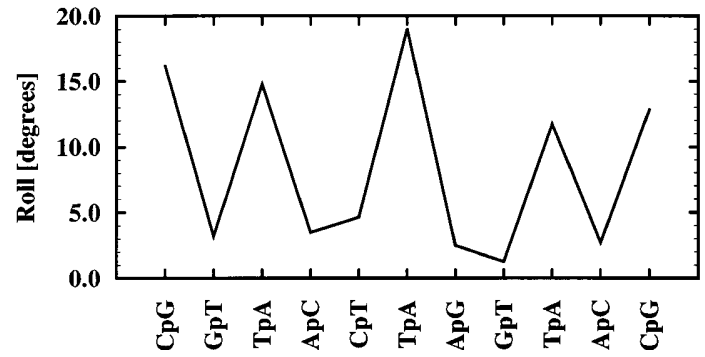

Fig. 2 Average roll parameters as a function of the DNA sequence calculated from the last nanosecond of the MD trajectory (0.4-1.4 ns) with Curves 5.3 (Lavery and Sklenar 1988)

model of DNA bending by electrostatic collapse around counterions (Rouzina and Bloomfield 1998; Shui et al. 1998; Hud et al. 1999). It is not possible, however, to distinguish between DNA bending as a result of sodium localisation in the major groove or vice versa. The increased roll at pyrimidine-purine steps, which causes the DNA to bend toward the major groove, has also been observed in simulations of A-tract DNA (Sanghani et al. 1996; Sprous et al. 1999). To further characterise the sodium ion distribution around the DNA, the phosphate-sodium radial distribution function $\left[g_{\mathrm{P}-\mathrm{Na}}(r)\right]$ was calculated from the trajectory (Fig. 3). Only a very small fraction $(<1 \%)$ of the sodium ions are directly bound to the phosphate groups, as can be seen from the very small peak at $0.37 \mathrm{~nm}$. The next peak of $g_{\mathrm{P}-\mathrm{Na}}(r)$ at $\sim 0.58 \mathrm{~nm}$ corresponds to the sodium ions in the DNA grooves. From the coordination number plotted in Fig. 3 (dashed line) a phosphate charge neutralisation of 0.76 is estimated at $\sim 0.7 \mathrm{~nm}$ from the DNA surface $(\sim 0.8 \mathrm{~nm}$ from the phosphate atom in Fig. 3). This is in very good agreement with Manning's polyelectrolyte condensation theory (Manning 1978). Analysis of other radial distribution functions revealed ion binding in the minor groove at the first ApC step. The binding occurred after $\sim 1.12 \mathrm{~ns}$ and the sodium ion kept its position for the remaining of the simulation up to $1.4 \mathrm{~ns}$. A snapshot of the bound ion taken at 1.14 ns is shown in Fig. 4. The sodium is coordinated by the two carbonyl groups of

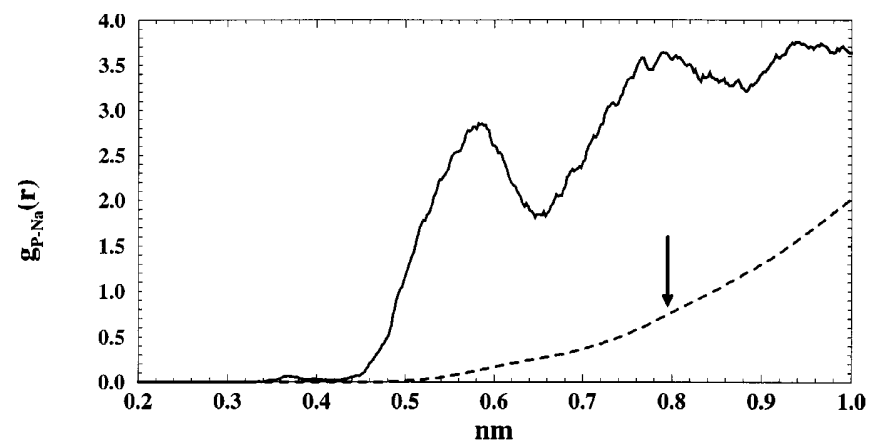

Fig. 3 Phosphate-sodium radial distribution function (plain line) and coordination number (dotted line) calculated from the last nanosecond of the MD trajectory $(0.4-1.4 \mathrm{~ns})$. The Manning's radius (Manning 1978), estimated from the coordination number value of 0.76 , is indicated by a vertical arrow

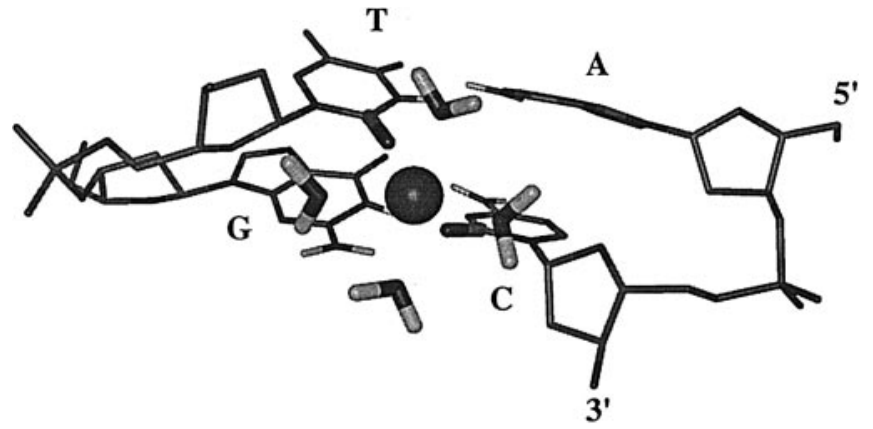

Fig. 4 Detail view of the sodium binding site in the major groove at the CpA step from a snapshot at $1.14 \mathrm{~ns}$. The sodium ion is coordinated by the carbonyl groups of Cyt and Thy and four water molecules. The figure was generated with VMD (Humphrey et al. 1996)

Cyt and Thy and four water molecules. A similar binding geometry at an ApT step was observed in a MD simulation of a DNA dodecamer (Young et al. 1997). Because of the limited time scale of this simulation, no conclusion can be drawn on the residence time of the bound ion.

The dynamics of the sodium counterions around DNA was investigated by calculating the average and maximum residence times within a $0.54 \mathrm{~nm}$ radius sphere around the phosphate oxygen atoms in the same manner as was done for the hydration study (Bonvin et al. 1998). The chosen radius corresponds to the first minimum of the phosphate oxygen-sodium radial distribution function (data not shown). The results are summarized in Table 2. The average residence time around phosphate groups was $46 \pm 38$ ps with a maximum of 345 ps. The localisation of the ions around the phosphate groups was further characterised by calculating a $B$-factor from the position of all sodium ions within the previously defined spheres around the phosphate oxygen atoms. The resulting average $B$-factor over all phosphate groups was $0.45 \pm 0.38 \mathrm{~nm}^{2}$, indicative of well-localised ions and consistent with the probability maps displayed in Fig. 1. For comparison, the average and maximum residence times of water molecules around the phosphate groups are $62 \pm 15 \mathrm{ps}$ and $500 \mathrm{ps}$, respectively, with a corresponding average $B$ factor of $1.1 \pm 1.0 \mathrm{~nm}^{2}$. While residing, on average, for similar periods of time around the phosphate groups, water molecules are much less localised than the sodium ions, as indicated by their high $B$-factors. The residence

Table 2 Residence times and $B$-factors of sodium ions and water molecules within a $0.54 \mathrm{~nm}$ radius sphere around the phosphate oxygen atoms

\begin{tabular}{llll}
\hline Molecule & $\begin{array}{l}\text { Average } \\
\text { residence } \\
\text { time }(\mathrm{ps})\end{array}$ & $\begin{array}{l}\text { Maximum } \\
\text { residence } \\
\text { time }(\mathrm{ps})\end{array}$ & $\begin{array}{l}B \text {-factors } \\
\left(\mathrm{nm}^{2}\right)\end{array}$ \\
\hline $\mathrm{Na}$ & $46 \pm 38$ & 345 & $0.46 \pm 0.38$ \\
$\mathrm{H}_{2} \mathrm{O}$ & $62 \pm 15$ & 500 & $1.10 \pm 1.00$ \\
\hline
\end{tabular}


time analysis indicates that, while condensed and well localised around the DNA, the sodium counterions still keep a highly dynamic character, which is again consistent with Manning's theory (Manning 1978). The few experimental data available on sodium ion dynamics around DNA in solution originate from NMR quadrupole relaxation studies (van Dijk et al. 1987; Groot et al. 1994) of ${ }^{23} \mathrm{Na}$. These studies indicate that at least two correlation times are required to characterise the spectral density function for ${ }^{23} \mathrm{Na}$ relaxation: a slow process in the nanosecond time range which can be correlated to the residence time of the ions around the DNA and disappears at the DNA duplex melting temperature, and a fast process correlated to the properties of the hydration water of the DNA. Unfortunately, correlation times can only be determined from experimental NMR data for the slow process, while the product of the correlation time and mean-squared quadrupolar coupling constant is obtained for the fast process (van Dijk et al. 1987; Groot et al. 1994). Characterising nanosecond processes by MD simulation will require order of magnitude increases in simulation time. The fast processes, however, are within the reach of such simulations. The dynamics of hydration water of this particular DNA sequence studied by MD simulations (Bonvin et al. 1998) has been shown to agree with NMR measurements (Sunnerhagen et al. 1998).

In conclusion, the picture of the localisation and dynamics of sodium counterions around this $16 \mathrm{mer}$ DNA sequence in solution agrees with previous theoretical and experimental studies of A-tract DNA sequences and fits the condensation model of Manning. A sequence- specific effect is seen at pyrimidine-purine steps where bending correlates with localised sodium probability, which would fit in the recently proposed electrostatic collapse bending model.

Acknowledgements The author thanks Prof. W.F. van Gunsteren, ETH Zurich, Switzerland, and Dr. P. Furrer, EPF Lausanne, Switzerland, for helpful discussions.

\section{References}

Arnott S, Campbell-Smith PJ, Chandrasekaran R (1976) Atomic coordinates and molecular conformations for DNA-DNA, RNA-RNA, and DNA-RNA helices. In: CRC handbook of biochemistry and molecular biology. CRC Press, Boca Raton, pp 411-422

Berendsen HJC, Postma JPM, Gunsteren WF van, Hermans J (1981) Interaction models for water in relation to protein hydration. In: Pullman B (ed) Intermolecular forces. Reidel, Dordrecht, pp 331-342

Berendsen HJC, Postma JPM, Gunsteren WF van, DiNola A, Haak JR (1984) Molecular dynamics with coupling to an external bath. J Chem Phys 81: 3684-3690

Bernstein FC, Koetzle TF, Williams GJ, Meyer EE Jr, Brice MD, Rodgers JR, Kennard O, Shimanouchi T, Tasumi, M (1987) The Protein Data Bank: a computer-based archival file for macromolecular structures. J Mol Biol 112: 535-542

Bonvin AMJJ, Sunnerhagen M, Otting G, Gunsteren WF van (1998) Water molecules in DNA recognition II: a molecular dynamics view of the structure and hydration of the trp operator. J Mol Biol 282: 859-873

Brünger AT (1993) X-PLOR version 3.1: a system for X-ray crystallography and NMR. Yale University Press, New Haven

DeLano WL, Brünger AT (1994) Helix packing in proteins: prediction and energetic analysis of dimeric, trimeric and tetrameric GCN4 coiled coil structures. Proteins Struct Funct Genet 20: $105-123$

Dijk L van, Gruwel MLH, Jesse W, de Bleijser J, Leyte JC (1987) Sodium ions and solvent nuclear relaxation results in aqueous solutions of DNA. Biopolymers 26: 261-284

Evans SV (1993) SETOR: hardware lighted three-dimensional solid model representations of macromolecules. J Mol Graphics 11: $134-138$

Hud NV, Sklenar V, Feigon J (1999) Localization of ammonium ions in the minor groove of DNA duplexes in solution and the origin of DNA A-tract bending. J Mol Biol 286: 651-660

Humphrey W, Dalke A, Schulten K (1996) VMD - visual molecular dynamics. J Mol Graphics 14: 33-38

Groot LCA, Marel JRC van der, Leyte JC (1994) ${ }^{23} \mathrm{Na}$ relaxation in isotropic and anisotropic liquid-crystalline DNA solutions. J Phys Chem 98: 2699-2705

Gunsteren WF van, Berendsen HJC (1990) Computer simulation of molecular dynamics: methodology, applications and perspectives in chemistry. Angew Chem Int Ed Engl 29: 992-1023

Gunsteren WF van, Billeter SR, Eising AA, Hünenberger PH, Krüger P, Mark AE, Scott WRP, Tironi IG (1996) Biomolecular simulation: the GROMOS96 manual and user guide. Hochschulverlag ETH Zürich, Zürich

Lavery R, Sklenar V (1988) The definition of generalized helicoidal parameters and of axis curvature for irregular nucleic acids. J Biomol Struct Dyn 6: 63-91

Lawson CL, Carey J (1993) Tandem binding in crystals of a trp repressor/operator half-site complex. Nature 366: 178-182

Lyubartsev AP, Laaksonen A (1998) Molecular dynamics simulations of DNA in solution with different counter-ions. J Biomol Struct Dyn 16: 579-592

Manning GS (1978) The molecular theory of polyelectrolyte solutions with application to the electrostatic properties of polynucleotides. Q Rev Biophys 11: 179-246

Rouzina I, Bloomfield VA (1998) DNA bending by small, mobile multivalent cations. Biophys J 74: 3152-3164

Ryckaert J-P, Ciccotti G, Berendsen HJC (1977) Numerical integration of the cartesian equations of motion of a system with constraints: molecular dynamics of n-alkanes. J Comput Phys 23: $327-341$

Sanghani SR, Zakrzewska K, Harvey SC, Lavery R (1996) Molecular modelling of $\left(\mathrm{A}_{4} \mathrm{~T}_{4} \mathrm{NN}\right)_{n}$ and $\left(\mathrm{T}_{4} \mathrm{~A}_{4} \mathrm{NN}\right)_{n}$ : sequence elements responsible for curvature. Nucleic Acids Res 24: 16321637

Scott WRP, Hünenberger PH, Tironi IG, Mark AE, Billeter SR, Fennen J, Torda AE, Huber T, Krüger P, van Gunsteren WF (1999) The GROMOS biomolecular simulation program package. J Phys Chem A 103: 3596-3607

Shui X, Sines CC, McFail-Isom L, VanDerveer D, Williams LD (1998) Structure of the potassium form of CGCGAATTCGCG: DNA deformation by electrostatic collapse around inorganic cations. Biochemistry 37: 16877-16887

Sprous D, Young MA, Beveridge DL (1999) Molecular dynamics studies of axis bending in $\mathrm{d}\left(\mathrm{G}_{5}-\left(\mathrm{GA}_{4} \mathrm{~T}_{4} \mathrm{C}_{2}\right)_{2} \mathrm{C}_{5}\right)$ and $\mathrm{d}\left(\mathrm{G}_{5-}\right.$ $\left.\left(\mathrm{GT}_{4} \mathrm{~A}_{4} \mathrm{C}\right)_{2}-\mathrm{C}_{5}\right)$ : effects of sequence polarity on DNA curvature. J Mol Biol 285: 1623-1632

Sunnerhagen M, Denisov VP, Venu K, Bonvin AMJJ, Carey J, Halle B, Otting G (1998) Water molecules in DNA recognition I: hydration lifetimes of the trp operator in solution measured by NMR spectroscopy. J Mol Biol 282: 847-858

Tironi IG, Sperb R, Smith PE, van Gunsteren WF (1995) A generalized reaction field method for molecular dynamics simulations. J Chem Phys 102: 5451-5459

Young MA, Ravishanker G, Beveridge DL (1997) A 5-nanosecond molecular dynamics trajectory for B-DNA: analysis of structure, motions and solvation. Biophys J 73: 2313-2336 